\title{
Decompressive craniectomy in traumatic brain injury-the discussion must continue
}

\author{
Teodor Svedung Wettervik ${ }^{1} \cdot$ Per Enblad $^{1}$
}

Received: 28 March 2018 / Accepted: 4 April 2018 / Published online: 16 April 2018

(C) Springer-Verlag GmbH Austria, part of Springer Nature 2018

Dear Editor,

We appreciate the encouraging and important comments in the letter concerning our study "Decompressive craniectomy in traumatic brain injury: indication and clinical outcome in a single centre" [3]. The question raised in the letter was "Is decision-making easier post RESCUE-ICP trial?"

The results of randomized clinical trials usually have a great impact of routine clinical management. We were afraid that the final word regarding the role of decompressive craniectomy was said after the disappointing results of the DECRA and RESCUE-ICP trials $[1,2]$. That was the reason why we felt it was important to describe the use of decompressive craniectomy in a clinical routine setting, using an escalated protocol in which both decompressive craniectomy and thiopental were included. We showed that the decisionmaking and use of decompressive craniectomy was quite different from the randomized clinical trials. When significant intracranial hypertension occurred, the patients were promptly treated with either thiopental or decompressive craniectomy, or both, subsequently in different orders. Decompressive craniectomy was also used as a primary procedure. The clin- ical outcome after 6 months appeared to be acceptable in this group of patients.

Our study does not provide definite evidence for which is the right decision but underlines that the final word not is said regarding the role of decompressive craniectomy after the DECRA and RESCUE-ICP trials. Additional randomized clinical trials are desirably more resembling the real-world scenario, although designing such studies is very difficult taking all factors into account in this complicated condition.

\section{References}

1. Cooper DJ, Rosenfeld JV, Murray L, Arabi YM, Davies AR, D’Urso $P$ et al (2011) Decompressive craniectomy in diffuse traumatic brain injury. N Engl J Med 364:1493-1502

2. Hutchinson PJ, Kolias AG, Timofeev IS, Corteen EA, Czosnyka M, Timothy J et al (2016) Trial of decompressive craniectomy for traumatic intracranial hypertension. N Engl J Med 375:1119-1130

3. Wettervik TS, Lenell S, Nyholm L, Howells T, Lewén A, Enblad P (2017) Decompressive craniectomy in traumatic brain injury: usage and clinical outcome in a single centre. Acta Neurochir:1-9
Teodor Svedung Wettervik

teodor.svedung-wettervik@neuro.uu.se

1 Department of Neuroscience, Section of Neurosurgery, Uppsala University Hospital, Uppsala University, SE-751

85 Uppsala, Sweden 